\title{
Corrected coupled-wave theory for non-slanted reflection gratings
}

\author{
L. Alberto Estepa*a ${ }^{*}$, Cristian Neipp ${ }^{\mathrm{a}}$, Jorge Francés ${ }^{\mathrm{a}}$, Andrés Márquez ${ }^{\mathrm{a}}$, Sergio Bleda ${ }^{\mathrm{a}}$, Manuel \\ Pérez-Molina ${ }^{a}$, Manuel Ortuño ${ }^{\text {a }}$, Sergi Gallego ${ }^{\text {a }}$ \\ ${ }^{a}$ Dpto. de Física, Ingeniería de Sistemas y Teoría de la Señal, Universidad de Alicante/Ctra. San \\ Vicente del Raspeig, San Vicente del Raspeig, Alicante, España E-03080;
}

\begin{abstract}
In this work we present an analysis of non-slanted reflection gratings by using a corrected Coupled Wave Theory which takes into account boundary conditions. It is well known that Kogelnik's Coupled Wave Theory predicts with great accuracy the response of the efficiency of the zero and first order for volume phase gratings, for both reflection and transmission gratings. Nonetheless, since this theory disregard the second derivatives in the coupled wave equations derived from Maxwell equations, it doesn't account for boundary conditions. Moreover only two orders are supposed, so when either the thickness is low or when high refractive index high are recorded in the element Kogelnik's Theory deviates from the expected results. In Addition, for non-slanted reflection gratings, the natural reflected wave superimpose the reflection order predicted by Coupled Wave theories, so the reflectance cannot be obtained by the classical expression of Kogelnik's Theory for reflection gratings. In this work we correct Kogelnik's Coupled Wave Theory to take into account these issues, the results are compared to those obtained by a Matrix Method, showing good agreement between both theories.
\end{abstract}

Keywords: Coupled Wave Theory, Holography, Diffraction grating, Volume phase grating

\section{INTRODUCTION}

The study of the interaction of electromagnetic radiation with diffractive elements has received much attention in the literature. In particular, several theoretical models have been proposed to accurately describe the behaviour of diffraction gratings of different kind. The attention posed on these structures is in part due to the fact that a sinusoidal diffraction grating is the simplest periodic structure that can be recorded on a photosensitive material. Therefore the basic problem in volume holography theory is to describe accurately the properties of this kind of structures ${ }^{1}$. A usual way to calculate the efficiencies of the different orders that propagate in the volume grating is to solve Maxwell equations for the case of an incident plane wave on a medium where the relative dielectric permittivity varies ${ }^{2}$. Although the idea seems clear and precise, in the literature there are a great number of models that allow solving the problem.

One of the most used models to solve the electric field in the periodic structure is the Coupled Wave (CW) theory ${ }^{1,2}$. The name of this method is directly related to the way the solution of the wave equations is obtained. The most representative aspect of the CW theory is that it assumes a continuous interchange of energy between the waves that propagate inside the grating. The first study to calculate the electric field inside a holographic dielectric grating using the coupled wave method was made by Kogelnik in $1969^{3}$. Kogelnik assumed that only two orders propagated in the hologram, orders zero and +1 , and obtained analytical solutions for the efficiencies of the first and zero order when a plane wave impinges on a diffraction grating with a sinusoidal variation of its electro-optical properties (relative dielectric permittivity and conductivity). The highly predictive character of the expressions derived by Kogelnik made his work one of the most cited by holographic researchers. Nonetheless Kogelnik's theory assumed some approximations that make it inaccurate for some cases, such as dielectric gratings that are not sinusoidal or for thin gratings (outside the Bragg regime). For these cases the Rigorous Coupled Wave (RCW) theory proposed by Moharam and Gaylord ${ }^{4}$ in 1982 has proven to be appropriated. Moharam and Gaylord proposed the method to solve rigorously (apart for the number of orders chosen for the numerical simulations) the differential coupled wave equations that emerge from the Helmoltz equation when the coupled wave theory is used.

The Rigorous Coupled Wave Theory has been applied with success to volume holograms and binary gratings ${ }^{5-10}$, photonic band structures ${ }^{11}$, diffractive lenses ${ }^{12}$, etc. And it is also the method that should be used to test the validity of the different approximations that have been done and are still doing in order to obtain analytical functions for the

Physical Optics, edited by Daniel G. Smith, Frank Wyrowski, Andreas Erdmann,

Proc. of SPIE Vol. 8171, 81710R - @ 2011 SPIE · CCC code: 0277-786X/11/\$18

doi: $10.1117 / 12.896877$

Proc. of SPIE Vol. $817181710 R-1$ 
efficiencies of the different orders that propagate in the hologram. Although exact predictions can be obtained by using the RCW it is still interesting to find analytical expressions in order to calculate the efficiency of the different orders that propagate inside the hologram. Analytical expressions give a deeper understanding of the physical processes than numerical solutions do. In addition, by direct inspection of the analytical expressions a clearer interpretation of how the different parameters influence in the efficiency of the different orders is got.

In this work we present a model based on Kogelnik's Coupled Wave equations to study the behavior of reflected and transmitted orders that propagate in a non-slanted reflection grating. The model is based on assuming more general boundary conditions than Kogelnik does, thus permitting a different strategy for correcting Kogelnik's expressions of diffraction and transmission efficiency when the grating is embedded in media with different refractive indexes. The results obtained by using this method are compared with those obtained by using a solution of the differential equation in terms of Mathieu functions, showing good agreement when either the diffraction grating is index-matched or it is not.

\section{CORRECTED COUPLED WAVE THEORY}

To start with we will derive the basic equations that govern the behaviour of the different orders that propagate inside a lossless reflection diffraction grating applying Kogelnik's Coupled Wave Theory.

We will start with the coupled equations derived by Kogelnik ${ }^{3}$ that describe the energy interchanged between order $0, \mathrm{R}$, and order +1 reflected, $\mathrm{S}$

$$
\begin{gathered}
c_{R} \frac{d R}{d z}+j \kappa S=0 \\
c_{S} \frac{d S}{d z}+j \vartheta S+j \kappa R=0
\end{gathered}
$$

where

$$
\kappa=\frac{\pi n_{1}}{\lambda}
$$

$n_{l}$ being the refractive index modulation and $\lambda$ the wavelength in air, the off-Bragg parameter is defined as:

$$
\vartheta=\frac{\beta^{2}-\sigma^{2}}{2 \beta}
$$

where $\beta$ is the modulus of the propagation vector of order zero inside the grating.

And $c_{R}$ and $c_{S}$ are the cosine of the angles formed by the propagation vectors of the zero order and that of the first order with respect to the normal of the grating:

$$
\begin{gathered}
c_{R}=\cos \theta_{1} \\
c_{S}=c_{R}+\frac{K}{\beta} \sin \varphi
\end{gathered}
$$

Where $\theta_{l}$ is the angle formed by the incident wave with the normal of the grating and $\varphi$ is the angle that the fringes of the grating form with the $\mathrm{z}$ axis.

The solutions of the coupled wave equations can be found in terms of exponentials as:

$$
\begin{aligned}
& R(z)=r_{1} \exp \gamma_{1} z+r_{2} \exp \gamma_{2} z \\
& S(z)=s_{1} \exp \gamma_{1} z+s_{2} \exp \gamma_{2} z
\end{aligned}
$$

where: 


$$
\gamma_{1,2}=-\frac{1}{2} j \frac{\vartheta}{c_{S}} \pm \frac{1}{2}\left[\left(-j \frac{\vartheta}{c_{S}}\right)^{2}-4 \frac{\kappa^{2}}{c_{R} c_{S}}\right]^{1 / 2}
$$

These results were derived by Kogelnik. Now by imposing the conditions $R(0)=1$ and $S(d)=0$, he found the expressions for the efficiency of the zero and first reflected order when a plane wave impinges onto the diffraction grating. Nonetheless, we will suppose a more general form of the boundary conditions to allow for the incidence of two waves with the directions of the zero and the first reflected order. Therefore we allow for the following conditions:

$$
\begin{aligned}
& R(0)=R_{0} \\
& S(d)=S_{0}
\end{aligned}
$$

Now substituting equations (7-8) into (1-2) and taking into account (10-11) the following system of linear equations can be obtained:

$$
\begin{gathered}
r_{1}+r_{2}=R_{0} \\
S_{1} \exp \gamma_{1} d+s_{2} \exp \gamma_{2} d=S_{0} \\
c_{R}\left(\gamma_{1} r_{1}+\gamma_{2} r_{2}\right)=-j \kappa\left(s_{1}+s_{2}\right) \\
c_{S}\left(\gamma_{1} s_{1} \exp \gamma_{1} d+\gamma_{2} s_{2} \exp \gamma_{2} d\right)=-j \vartheta S_{0}-j \kappa\left(r_{1} \exp \gamma_{1} d+r_{2} \exp \gamma_{2} d\right)
\end{gathered}
$$

Solving the system for $r_{1}, r_{2}, s_{1}$ and $s_{2}$ and using the equations $(1-2,7-8,10-11)$ the amplitudes of the zero and first reflected orders can be found. In particular the values obtained for $s_{1}$ and $s_{2}$ are:

$$
\begin{gathered}
S_{1}=S_{0} \frac{\kappa^{2}\left(e^{\gamma_{1} d}-e^{\gamma_{2} d}\right)+c_{R} e^{\gamma_{2} d}\left(c_{S}\left(\gamma_{1}-\gamma_{2}\right) \gamma_{2}+j\left(\gamma_{1}-\gamma_{2}\right) \vartheta+R_{0} / S_{0} \kappa\left(e^{\gamma_{2} d} \gamma_{1}-e^{\gamma_{1} d} \gamma_{2}\right)\right.}{\kappa^{2}\left(e^{\gamma_{1} d}-e^{\gamma_{2} d}\right)^{2}-c_{R} c_{S} e^{\left(\gamma_{1}+\gamma_{2}\right) d}\left(\gamma_{1}-\gamma_{2}\right)^{2}} \\
S_{2}=-S_{0} \frac{\kappa^{2}\left(e^{\gamma_{1} d}-e^{\gamma_{2} d}\right)+c_{R} e^{\gamma_{2} d}\left(c_{S}\left(\gamma_{1}-\gamma_{2}\right) \gamma_{1}+j\left(\gamma_{1}-\gamma_{2}\right) \vartheta+R_{0} / S_{0} \kappa\left(e^{\gamma_{2} d} \gamma_{1}-e^{\gamma_{1} d} \gamma_{2}\right)\right.}{\kappa^{2}\left(e^{\gamma_{1} d}-e^{\gamma_{2} d}\right)^{2}-c_{R} c_{S} e^{\left(\gamma_{1}+\gamma_{2}\right) d}\left(\gamma_{1}-\gamma_{2}\right)^{2}}
\end{gathered}
$$

Now if $t_{i j}$ and $r_{i j}$ are the Fresnel coefficients, from medium i to medium $\mathrm{j}$ :

$$
\begin{aligned}
t_{i j} & =\frac{2 n_{i} \cos \theta_{i}}{n_{i} \cos \theta_{i}+n_{j} \cos \theta_{j}} \\
r_{i j} & =\frac{n_{i} \cos \theta_{i}-n_{j} \cos \theta_{j}}{n_{i} \cos \theta_{i}+n_{j} \cos \theta_{j}}
\end{aligned}
$$

And $S(d)$ is the amplitude of the reflected order obtained by imposing $R_{0}=t_{a g}$ and $S_{0}=0$ in equations $(8,16-17)$. The amplitude of the reflected wave in air can be obtained as:

$$
S_{a i r}=\left\lfloor r_{a g}+t_{g a} S(d)\right\rfloor
$$

Where the subscript, $a$, means air and, $g$, grating.

The efficiency of the reflected wave is finally obtained as:

$$
\eta=S_{a i r} S^{*}{ }_{\text {air }}
$$




\section{SOLUTION IN TERMS OF MATHIEU FUNCTIONS}

In this section a solution for the efficiency of the reflected order for a non-slanted reflection grating (Figure 1) is obtained in terms of Mathieu functions.

Consider a plane electromagnetic wave incident onto a periodic non-magnetic medium, which dielectric constant varies in form:

$$
\varepsilon_{r}=\varepsilon_{r 0}+\varepsilon_{r 1} \cos (K z)
$$

The treatment is done only for TE polarization, but can be extended to TM polarization. In this case the function E (z) for the electric field inside the medium verifies the following differential equation:

$$
\frac{d^{2} E}{d z^{2}}+\left[k_{0}{ }^{2}\left(\varepsilon_{r 0}+\varepsilon_{r 1} \cos (K z)\right)-K_{x}{ }^{2}\right] E=0
$$

Where:

$$
k_{0}=\frac{2 \pi}{\lambda}
$$

Being $\lambda$ the wavelength in vacuum.

If $\theta_{1}$ is the angle of incidence and $\theta_{2}$ is the angle between the wave vector and the normal to the substrate of refractive index $n_{2}$, then the following parameters can be defined:

$$
\begin{gathered}
K_{x}=n_{1}(\omega / c) \sin \theta_{1}=n_{2}(\omega / c) \sin \theta_{2} \\
q_{1}=n_{1}(\omega / c) \cos \theta_{1} \\
q_{2}=n_{2}(\omega / c) \cos \theta_{2}
\end{gathered}
$$

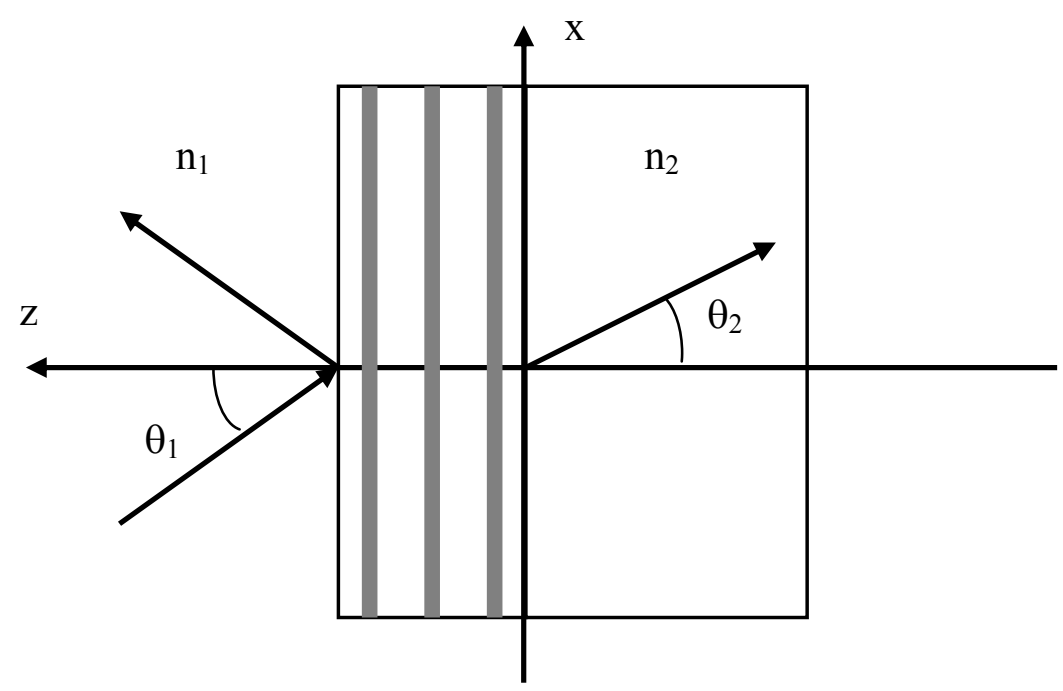

Figure 1.- Nonslanted reflection grating

The electric field in the first medium can be expressed as the superposition of an incident wave and a reflected wave of the form: 


$$
E^{I}(z)=\exp \left(j q_{1} z\right)+r_{s} \exp \left(-j q_{1} z\right)
$$

While in the second medium only the transmitted wave exist:

$$
E^{I I}(z)=t_{s} \exp \left(j q_{1} z\right)
$$

Now suppose that $\mathrm{f}(\mathrm{z})$ is a solution of the differential equation with a unit amplitude transmitted wave:

$$
E^{I I}(z)=\exp \left(j q_{1} z\right)
$$

with initial conditions:

$$
\begin{gathered}
E(0)=1 \\
\frac{d E}{d z}(0)=j q_{2}
\end{gathered}
$$

The boundary conditions in $\mathrm{z}=\mathrm{d}$ imply:

$$
\begin{gathered}
E(d)=\exp \left(j q_{1} d\right)+r_{s} \exp \left(-j q_{1} d\right) \\
\frac{d E}{d z}(d)=j q_{1} \exp \left(j q_{1} d\right)-j q_{1} r_{s} \exp \left(-j q_{1} d\right)
\end{gathered}
$$

Given the linearity of Maxwell's equations we have:

$$
\begin{gathered}
t_{s} f(d)=\exp \left(j q_{1} d\right)+r_{s} \exp \left(-j q_{1} d\right) \\
t_{s} \frac{d f}{d z}(d)=j q_{1} \exp \left(j q_{1} d\right)-j q_{1} r_{s} \exp \left(-j q_{1} d\right)
\end{gathered}
$$

From equations (35) and (36) we can obtain the amplitudes of the reflected and transmitted waves based on the solution $\mathrm{f}(\mathrm{z})$ with initial conditions (31) and (32):

$$
\begin{gathered}
t_{s}=\frac{2 q_{1} \exp \left(j q_{1} d\right)}{-j \frac{d f}{d z}(d)+q_{1} f(d)} \\
r_{s}=\frac{\frac{d f}{d z}(d)-j q_{1} f(d)}{\frac{d f}{d z}(d)+j q_{1} f(d)} \exp \left(2 j q_{1} d\right)
\end{gathered}
$$

It is now necessary to obtain a solution of the differential equation with initial conditions (31) and (32).

In this case the differential equation can be solved in terms of Mathieu functions. If we call

$$
a=\frac{4\left(-K_{x}{ }^{2}+k_{0}{ }^{2} \varepsilon_{r 0}\right)}{K^{2}}
$$

and

$$
q=\frac{-2 k_{0}{ }^{2} \varepsilon_{r 1}}{K^{2}}
$$

The function $\mathrm{f}(\mathrm{z})$, solution of the differential equation (23) with initial conditions (31-32) has the form: 


$$
\begin{aligned}
f(z)= & \frac{\operatorname{sm}(a, q, K z / 2)\left(2 j q_{2} c m(a, q, 0)-K \cdot \operatorname{cmp}(a, q, 0)\right)}{K(c m p(a, q, 0) \operatorname{sm}(a, q, 0)-c m(a, q, 0))}+ \\
& +\frac{c m(a, q, K z / 2)\left(-2 j q_{2} \operatorname{sm}(a, q, 0)-K \cdot \operatorname{smp}(a, q, 0)\right)}{K(c m p(a, q, 0) \operatorname{sm}(a, q, 0)-c m(a, q, 0))}
\end{aligned}
$$

And its derivative:

$$
\begin{aligned}
f^{\prime}(z)= & \frac{c m p(a, q, K z / 2)\left(-2 j q_{2} \operatorname{sm}(a, q, 0)+K \cdot \operatorname{smp}(a, q, 0)\right)}{2 \operatorname{cmp}(a, q, 0) \operatorname{sm}(a, q, 0)-2 \operatorname{smp}(a, q, 0) \operatorname{cm}(a, q, 0)}+ \\
& +\frac{\operatorname{smp}(a, q, K z / 2)\left(2 j q_{2} \operatorname{sm}(a, q, 0)-K \cdot \operatorname{cmp}(a, q, 0)\right)}{2 \operatorname{cmp}(a, q, 0) \operatorname{sm}(a, q, 0)-2 \operatorname{smp}(a, q, 0) \operatorname{cm}(a, q, 0)}
\end{aligned}
$$

Where $\mathrm{cm}(a, q, z)$ is the even Mathieu function $\operatorname{sm}(a, q, z)$ the odd Mathieu function, $\operatorname{cmp}(a, q, z)$ and $\operatorname{smp}(a, q, z)$ the corresponding derivatives.

\section{RESULTS AND DISCUSSION}

Now we want to compare the results obtained by using the corrected Coupled Wave Theory with those obtained using the solution in terms of Mathieu function. We will also show the results obtained using Kogelnik's Coupled Wave Theory corrected only for Fresnel losses. Firstly, we will study a non slanted reflection grating $12,5 \mu \mathrm{m}$ thick with a period $\Lambda$ of $0,25 \mu \mathrm{m}$ and a refractive index modulation $\Delta n=0,015$, a plane wave was supposed to impinge onto the grating with a wavelength of $0,633 \mathrm{~mm}$. In this case, it will be assumed that the grating was index matched with the surrounding media. Figure 2 shows the efficiency of the first reflected order as a function of the angle of incidence for a non-slanted reflection grating with an average refractive index equal to that of the surrounding media. As can be seen the results obtained by using Kogelnik's expression of the diffraction efficiency and those obtained using the solution in terms of Mathieu functions agree. It should be also indicated that the results of the corrected Coupled Wave Theory are the same to those obtained using only Kogelnik. Therefore, it is clear that when calculating the diffraction efficiency inside the grating there are no differences between the three approaches presented.

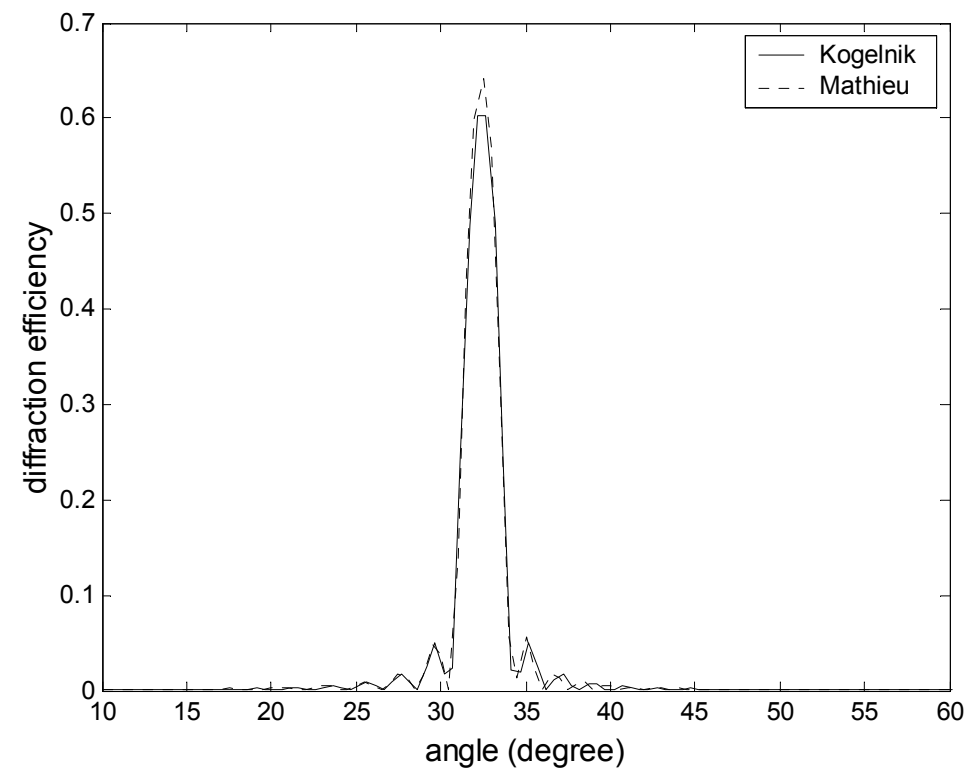

Figure 2.- Diffraction efficiency for a non-slanted reflection grating $12,5 \mu \mathrm{m}$ thick with a period $\Lambda$ of $0,25 \mathrm{~mm}$ and a refractive index modulation $\Delta n=0,015$. The grating was index-matched to the surrounding media. Koglenik's theory: continuous line; Mathieu solution: discontinuous line 
Things are different when there are differences between the refractive indexes of the periodic medium and the surrounding ones. In this case correcting Kogelnik's expression of the diffraction efficiency with Fresnel coefficients it is not suffice to achieve correct results. Figure 3 shows the efficiency of the first reflected order as a function of the angle for a non-slanted reflection grating $11 \mu \mathrm{m}$ thick with a period $\Lambda$ of $0,22 \mu \mathrm{m}$ and a refractive index modulation $\Delta n=$ 0,015 , the average refractive index of the grating was supposed to be $n=1,5$, whereas that of the first medium was supposed to be 1,0. The results are presented for Mathieu solution and Kogelnik's Theory, and as can be seen there are clear differences between both curves. The most representative is the fact that the angle with maximum diffraction efficiency is not the same for both models. Moreover the value of the maximum diffraction efficiency is also different for both cases. It is clear, then, that correcting Kogelnik's expression for Fresnel losses is not suffice to obtain accurate results. A more precise correction is performed by using the proposed corrected Coupled Wave Theory, figure 4 shows the efficiency of the first reflected order for the same diffraction grating, in this case with results obtained by the proposed model and the Mathieu solution. As can be seen only slight differences exist for both methods, but there is coincidence of the angles for maximum diffraction efficiency and maximum values of the efficiency.

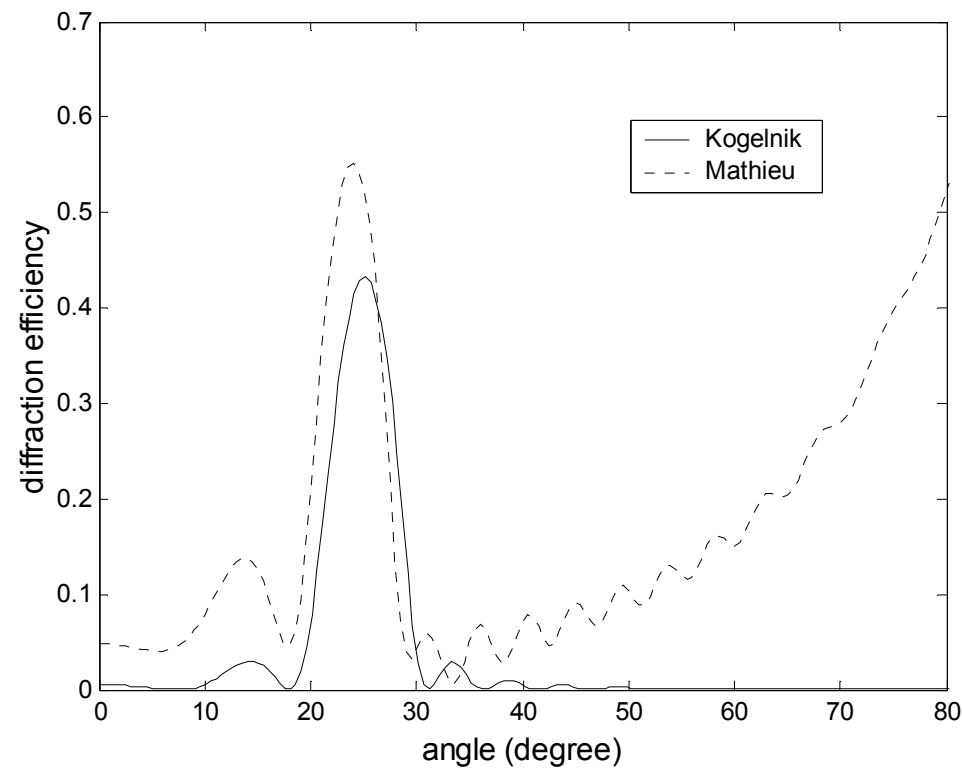

Figure 3.- Diffraction efficiency for a non-slanted reflection grating $11 \mu \mathrm{m}$ thick thick with a period $\Lambda$ of $0,22 \mu \mathrm{m}$ and a refractive index modulation $\Delta n=0,015$. The average refractive index of the grating was supposed to be 1,5 . Koglenik's theory: continuous line; Mathieu solution: discontinuous line

If the simulations are performed for a reflection grating with different parameters the same phenomena are observed. Figure 5 shows the efficiency of the first reflected order as a function of the angle for a non-slanted reflection grating $12,5 \mu \mathrm{m}$ thick with a period $\Lambda$ of $0,25 \mathrm{~mm}$ and a refractive index modulation $\Delta n=0,015$, results obtained by Mathieu solution and Kogelnik's Theory are presented. Whereas figure 6 show the results obtained by using corrected Coupled Wave Theory and those obtained in terms of Mathieu functions. As in the previous analyzed case the model based only in correcting Kogelnik's expression of the diffraction efficiency for Fresnel losses deviate from those obtained using corrected Coupled Wave Theory and Mathieu solution, demonstrating that in the case of non-slanted reflection gratings it is necessary to use more precise correction strategies of Kogelnik's Theory. 


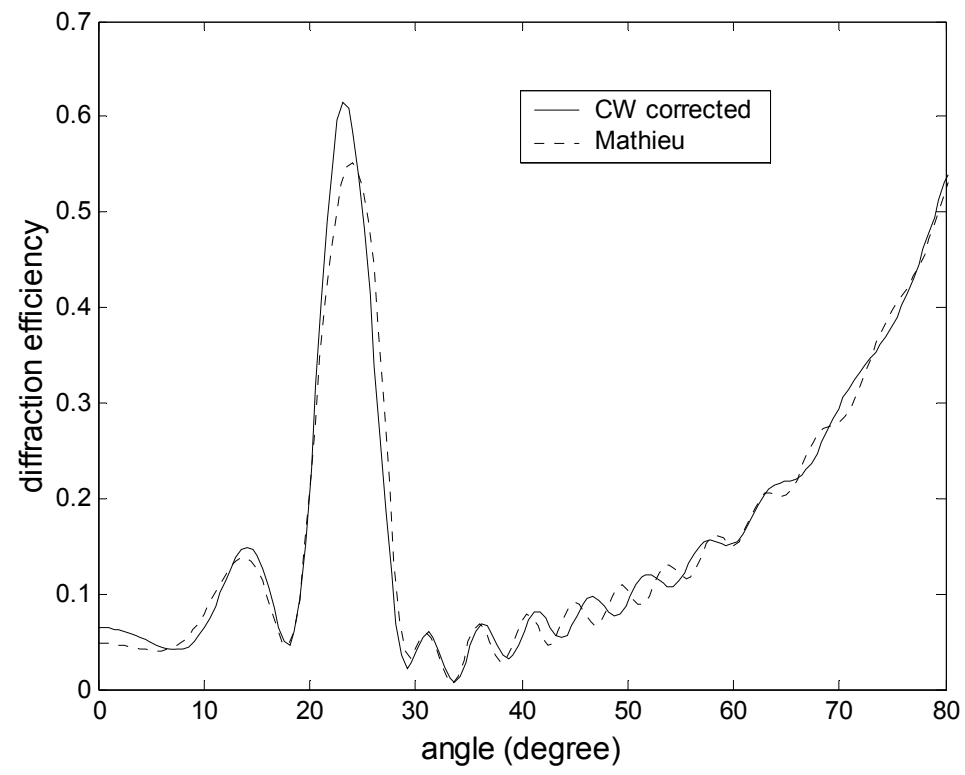

Figure 4.- Diffraction efficiency for a non-slanted reflection grating $11 \mu \mathrm{m}$ thick thick with a period $\Lambda$ of $0,22 \mu \mathrm{m}$ and a refractive index modulation $\Delta n=0,015$. The average refractive index of the grating was supposed to be 1,5 . Corrected Coupled Wave Theory: continuous line; Mathieu solution: discontinuous line

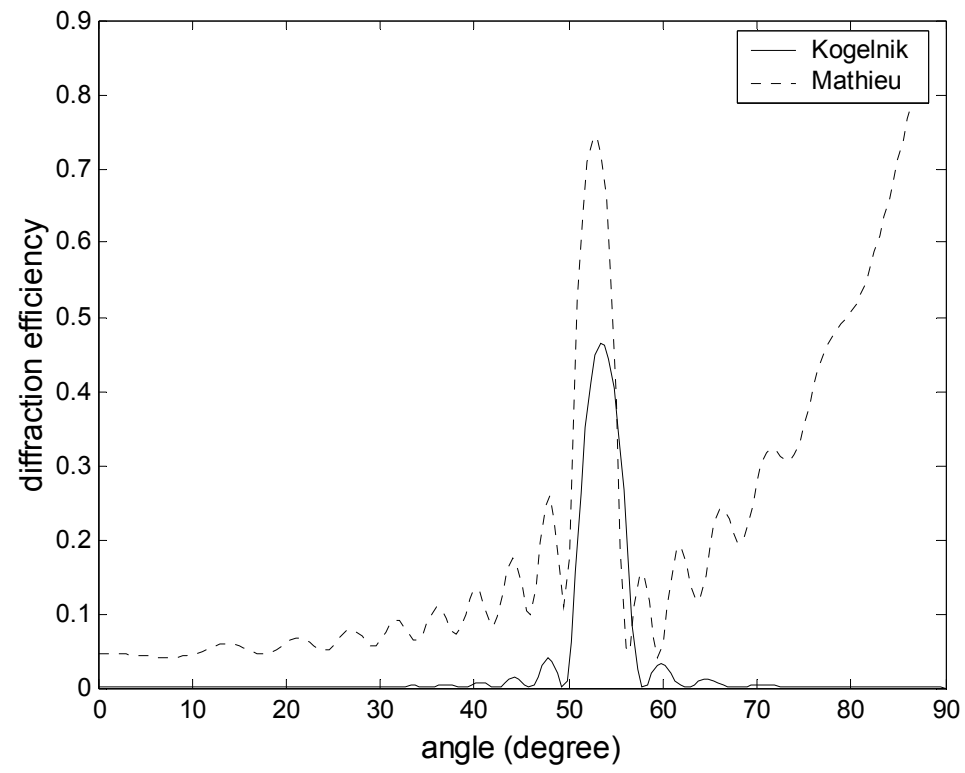

Figure 5.- Diffraction efficiency for a non-slanted reflection grating $12,5 \mu \mathrm{m}$ thick thick with a period $\Lambda$ of $0,25 \mu \mathrm{m}$ and a refractive index modulation $\Delta n=0,015$. The average refractive index of the grating was supposed to be 1,5 . Koglenik's theory: continuous line; Mathieu solution: discontinuous line. 


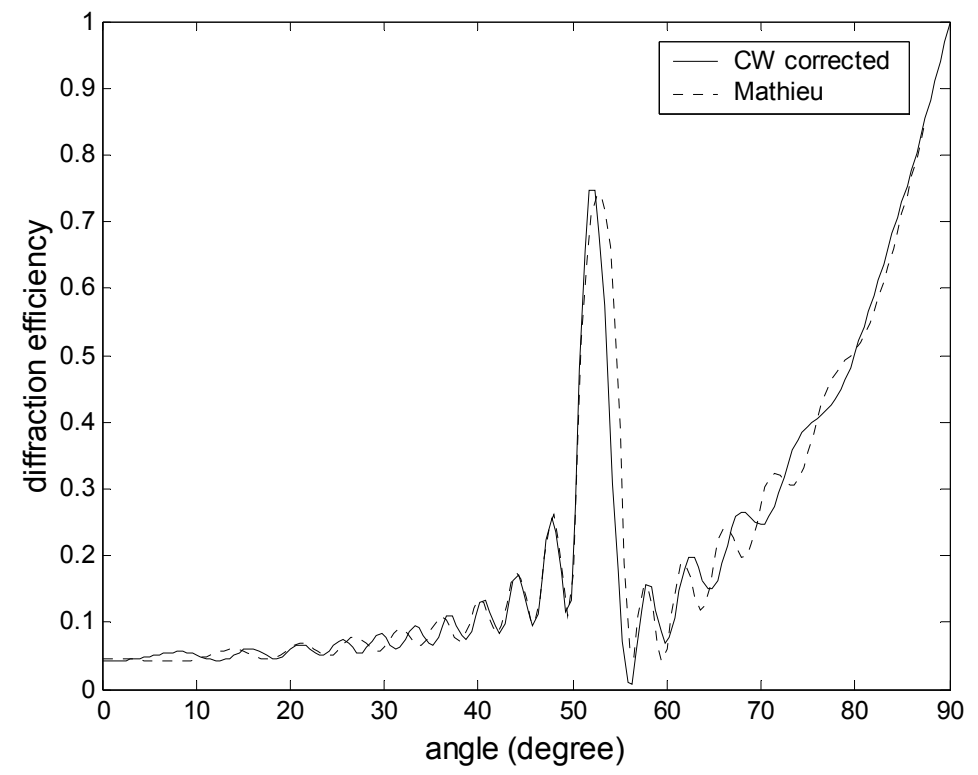

Figure 6.- Diffraction efficiency for a non-slanted reflection grating $12,5 \mu \mathrm{m}$ thick thick with a period $\Lambda$ of $0,25 \mu \mathrm{m}$ and a refractive index modulation $\Delta n=0,015$. The average refractive index of the grating was supposed to be 1,5. Corrected Coupled Wave Theory: continuous line; Mathieu solution: discontinuous line.

\section{CONCLUSIONS}

In this work a corrected Coupled Wave Theory is presented in order to analyze non-slanted reflection gratings. The model is based on Coupled Wave equations obtained by Kogelnik, but in this case different boundary conditions were imposed to obtain more general expressions of the amplitudes of the transmitted and reflected orders. An incidence wave and a reflected one were supposed to impinge onto the grating with arbitrary amplitudes, what allows a better correction strategy when the average refractive index of the grating is different from those of the surrounding media. In addition an alternative solution of the differential equation that governs the behavior of the electromagnetic radiation inside a reflection grating is described. This method permits obtaining the efficiency of the transmitted and the reflected order in terms of Mathieu functions. Finally the results obtained by using Kogelnik's Theory corrected for Fresnel losses, by using the corrected Coupled Wave Theory and the solution in terms of Mathieu functions are compared. The simulations made demonstrate that when the grating is index matched the three methods yield to the same results, but when this is not the case corrected Coupled Wave Theory yields to more accurate results than the correction of Kogelnik's expressions to take into account Fresnel losses.

\section{ACKNOWLEDGEMENTS}

This work was supported by the "Ministerio de Ciencia e Innovación" of Spain under projects FIS2008-05856-C02-01 and FIS2008-05856-C02-02, and by the "Generalitat Valenciana" of Spain under project PROMETEO/2011/021.

\section{REFERENCES}

[1] Solymar, L. and Cooke, D. J., [Volume Holography and Volume Gratings], Academic, London (1981).

[2] Syms, R. R. A., [Practical Volume Holography], Clarendon Press, Oxford (1990).

[3] Kogelnik, H., "Coupled wave theory for thick hologram gratings", Bell Syst. Tech. J. 48, 2909-2947 (1969).

[4] Moharam, M. G. and Gaylord, T. K., "Rigurous coupled-wave analysis of planar-grating diffraction", J. Opt. Soc. Am. 71, 811-818 (1981). 
[5] Moharam, M. G. and Gaylord, T. K., "Rigurous coupled-wave analysis of planar-grating diffraction- E-mode polarization and losses", J. Opt. Soc. Am. 73, 451-455 (1983).

[6] Moharam, M. G. and Gaylord, T. K., "Three dimensional vector coupled-wave analysis of planar-grating diffraction", J. Opt. Soc. Am. 73, 1105-1112 (1983).

[7] Gaylord, T. K. and Moharam, M. G., "Analysis and applications of optical diffraction by gratings", Proc. IEE 73, 894-937 (1985).

[8] Moharam, M. G. and Gaylord, T. K., "Rigurous coupled-wave analysis of metallic surface-relief gratings", J. Opt. Soc. Am. A 3, 1780-1796 (1986).

[9] Moharam, M. G., Grann, E. B., Pommet, D. A. and Gaylord, T. K., "Formulation for stable and efficienct implementation of the rigurous coupled-wave analysis of binary gratings", J. Opt. Soc. Am. A 12, 1068-1076 (1995).

[10] Kamiya, N., "Rigorous coupled-wave analysis for practical planar dielectric gratings: 1. Thickness-changed holograms and some characteristics of diffraction efficiency", Appl. Opt. 37, 5843-5853 (1998).

[11]Dansas, P. and Paraire, N., "Fast modelling of photonic bandgap structures by use of a diffraction-grating approach", J. Opt. Soc. Am. A 15, 1586-1598 (1998).

[12] Chang, N. Y. and Juo, C. J., "Algorithm based on rigorous coupled-wave analysis for diffractive optical element design”, J. Opt. Soc. Am . A 18, 2491-2501 (2001). 